Keywords: pancreatic cancer; Mycobacterium obuense; phase II; advanced pancreatic ductal adenocarcinoma; immunotherapy; gemcitabine; IMM-101; immunomodulator

\title{
Randomised, open-label, phase II study of gemcitabine with and without IMM-101 for advanced pancreatic cancer
}

Angus G Dalgleish ${ }^{*}, 1$, Justin Stebbing ${ }^{2}$, Douglas JA Adamson ${ }^{3}$, Seema Safia Arif ${ }^{4}$, Paolo Bidoli ${ }^{5}$, David Chang 6 , Sue Cheeseman ${ }^{7}$, Robert Diaz-Beveridge ${ }^{8}$, Carlos Fernandez-Martos ${ }^{9}$, Rob Glynne-Jones ${ }^{10}$, Cristina Granetto ${ }^{11}$, Bartomeu Massuti ${ }^{12}$, Karen McAdam ${ }^{13}$, Raymond McDermott ${ }^{14}$, Andrés J Muñoz Martín ${ }^{15}$, Demetris Papamichael ${ }^{16}$, Roberto Pazo-Cid ${ }^{17}$, Jose M Vieitez ${ }^{18}$, Alberto Zaniboni ${ }^{19}$, Kevin J Carroll ${ }^{20}$, Shama Wagle ${ }^{20}$, Andrew Gaya ${ }^{21}$ and Satvinder S Mudan ${ }^{22}$

${ }^{1}$ Cancer Vaccine Institute, St George's University of London, London, UK; ${ }^{2}$ Department of Oncology, Imperial College, Hammersmith Hospital, London, UK; ${ }^{3}$ Department of Oncology, Ninewells Hospital, Dundee, UK; ${ }^{4}$ Velindre Cancer Centre, Cardiff, UK; ${ }^{5}$ Department of Oncology, Azienda Ospedaliera San Gerardo, Monza, Italy; ${ }^{6}$ Department of General Surgery, Royal Blackburn Hospital, Blackburn, UK; ${ }^{7}$ Department of Oncology, Bradford Teaching Hospitals NHS Foundation Trust, Bradford, UK; ${ }^{8}$ Médico Adjunto de Oncología Médica, Hospital La Fe de Valencia, Valencia, Spain; ${ }^{9}$ Instituto Valenciano de Oncologia, Valencia, Spain; ${ }^{10}$ Mount Vernon Cancer Centre, Northwood, UK; ${ }^{11}$ Medical Oncology, Azienda Ospedaliera Santa Croce e Carle, Cuneo, Italy; ${ }^{12}$ Ensayos Clínicos Oncología, Hospital General Universitario de Alicante, Alicante, Spain; ${ }^{13}$ Oncology Department, Peterborough and Stamford Hospitals NHS Trust, Peterborough, UK; ${ }^{14}$ Medical Oncology, St Vincent's University Hospital and The Adelaide and Meath Hospital, Dublin, Republic of Ireland; ${ }^{15}$ Gastrointestinal Cancer Unit, Hospital General Universitario Gregorio Marañón, Madrid, Spain; ${ }^{16}$ Department of Medical Oncology, Bank of Cyprus Oncology Centre, Nicosia, Cyprus; ${ }^{17}$ Servicio de Oncología Médica, Hospital Miguel Servet, Zaragoza, Spain; ${ }^{18}$ Area and Neuroendocrine Tumors Gastrointestinal Medical Oncology, Hospital Central de Asturias, Asturias, Spain; ${ }^{19}$ Oncology Department, Fondazione Poliambulanza, Brescia, Italy; ${ }^{20}$ TranScrip Partners LLP, Reading, UK; ${ }^{21}$ Clinical Oncology, Guy's \& St Thomas' Hospitals NHS Trust, London, UK and ${ }^{22}$ St George's University of London, Imperial College, London and The Royal Marsden Hospital, London, UK

Background: Immune Modulation and Gemcitabine Evaluation-1, a randomised, open-label, phase II, first-line, proof of concept study (NCT01303172), explored safety and tolerability of IMM-101 (heat-killed Mycobacterium obuense; NCTC 13365) with gemcitabine (GEM) in advanced pancreatic ductal adenocarcinoma.

Methods: Patients were randomised (2:1) to IMM-101 (10 $\mathrm{mg} \mathrm{ml}^{-1}$ intradermally) + GEM (1000 $\mathrm{mg} \mathrm{m}^{-2}$ intravenously; $\left.n=75\right)$, or GEM alone $(n=35)$. Safety was assessed on frequency and incidence of adverse events (AEs). Overall survival (OS), progressionfree survival (PFS) and overall response rate (ORR) were collected.

Results: IMM-101 was well tolerated with a similar rate of AE and serious adverse event reporting in both groups after allowance for exposure. Median OS in the intent-to-treat population was 6.7 months for IMM-101 + GEM v 5.6 months for GEM; while not significant, the hazard ratio (HR) numerically favoured IMM-101 + GEM (HR, $0.68(95 \% \mathrm{Cl}, 0.44-1.04, P=0.074)$. In a pre-defined metastatic subgroup (84\%), OS was significantly improved from 4.4 to 7.0 months in favour of IMM-101 + GEM (HR, $0.54,95 \% \mathrm{Cl} 0.33-0.87, P=0.01)$.

Conclusions: IMM-101 with GEM was as safe and well tolerated as GEM alone, and there was a suggestion of a beneficial effect on survival in patients with metastatic disease. This warrants further evaluation in an adequately powered confirmatory study.

*Correspondence: Professor AG Dalgleish; Email: dalgleis@sgul.ac.uk

Received 31 March 2016; revised 22 June 2016; accepted 22 July 2016; published online 6 September 2016

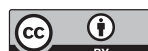

(c) 2016 Cancer Research UK. All rights reserved 0007-0920/16

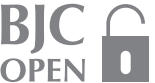


Only $18 \%$ of patients with advanced pancreatic ductal adenocarcinoma (PDAC) remain alive at 1 year, and $4 \%$ at 5 years (Hidalgo et al, 2015). Survival for metastatic disease is more dismal. Realworld studies report the overall median survival from diagnosis to be 4.6 months; in patients with metastatic cancer the median survival ranges between 2.8 and 5.7 months (Carrato et al, 2015).

When IMAGE-1 was set up, gemcitabine (GEM) was the standard of care for advanced PDAC (Burris et al, 1997; Network; Seufferlein et al, 2012) and at that time it was widely used as the comparator arm in clinical trials for this disease. Over the past 5 years, FOLFIRINOX, as well as GEM + nab-paclitaxel (Abraxane), have entered the clinical arena, mainly for patients with a good performance status because they increase toxicity significantly (Conroy et al, 2011; Von Hoff et al, 2013). The combination of nab-paclitaxel + GEM demonstrated clinical benefit in the firstline treatment of pancreatic cancer (Von Hoff et al, 2013) and was subsequently approved for the first-line treatment of metastatic adenocarcinoma of the pancreas. However, its use in clinical practice has experienced setbacks in Europe, for instance, in 2015, NICE (the National Institute for Health and Care Excellence) did not approve the use of nab-paclitaxel in combination with GEM, but it maintained the recommendation for GEM as the first-line treatment of advanced pancreatic cancer (NICE, 2001; NICE, 2015). The lack of reimbursement for nab-paclitaxel also limits use of this combination in the Benelux countries, Ireland and Eastern Europe. Despite the recent advances of FOLFIRINOX and nabpaclitaxel + GEM in the treatment of pancreatic cancer, the majority of pancreatic cancer patients $(76 \%)$ do not receive either of these regimens as first-line treatment (Braiteh et al, 2016). Therefore, an unmet need remains for therapies that confer meaningful survival advantages without additional toxicity.

Immunotherapy is effective in treating many cancers (Tempero et al, 2012), although success in PDAC is limited (Gunturu et al, 2013; Pico de Coana et al, 2015). Extended survival after secondline treatment with the therapeutic vaccine GVAX and CRS-207 (live-attenuated Listeria monocytogenes) has been reported after the treatment with low-dose cyclophosphamide (Le et al, 2015).

IMM-101 is a systemic immune modulator containing heatkilled Mycobacterium obuense (NCTC 13365). Results from in vivo and ex vivo non-clinical studies suggest that IMM-101 modulates the innate and adaptive immune systems, in response to cancer. IMM-101 acts on cells of the innate immune system, such as $\gamma \delta$ T-cells, granulocytes, and antigen-presenting cells, by interaction with a number of receptors (PAMPs-PRR; Fowler et al, 2011) (Bazzi et al, 2015). Activation of these cells is known to have a cytotoxic effect against tumours. Furthermore, it is proposed that IMM-101 restores Type-1 response, influences cytotoxic cell immune function and may downregulate Type 2 response. This is of significance because pancreatic cancer has been associated with a $\mathrm{T}_{\mathrm{h}} 2$ bias (Wormann et al, 2014).

In a phase I clinical study, IMM-101 was safe and well tolerated at the three escalating doses used in patients with melanoma (Stebbing et al, 2012). Therefore, IMAGE-1 was designed as a proof of concept (POC), phase II study primarily to explore the safety and tolerability of IMM-101 in combination with GEM vs GEM alone as first-line treatment in advanced PDAC. In addition, the study would provide some insight into the potential effects of treatment on the clinical signs and symptoms of disease including overall survival (OS), progressive-free survival (PFS) and overall response rate (ORR).

\section{MATERIALS AND METHODS}

Study design and patients. This open-label, phase II trial was conducted at 20 institutions in 5 countries (Cyprus, Ireland, Italy, Spain, UK). Eligible patients were age $\geqslant 18$ years, had confirmed inoperable PDAC (with or without metastatic disease), measurable lesions at $\geqslant 1$ site not previously irradiated, and WHO performance status (PS) $0-2$. Other inclusion criteria included serum albumin $\geqslant 26 \mathrm{gl}^{-6}$, C-reactive protein $(\mathrm{CRP}) \leqslant 70 \mathrm{mgl}^{-1}$, and life expectancy $>3$ months from randomisation. Exclusion criteria included prior PDAC chemotherapy, radiotherapy within 6 weeks of screening and chronic use of corticosteroids within 2 weeks of first study drug. The study was undertaken in compliance with the Declaration of Helsinki Principles and applicable local-regional regulations. The study protocol, the patient information leaflet and informed consent form were reviewed and approved by an Independent Ethics Committee/Institutional Review Board. All patients provided written informed consent. Patients were randomly assigned in a 2:1 ratio to receive IMM-101+ GEM or GEM alone by Interactive Response Technology. Randomisation was stratified according to disease extent and WHO PS, by computer generated block randomisation methods.

Procedures. In both groups, GEM was administered intravenously at $1000 \mathrm{mg} \mathrm{m}^{-2}$ over 30 min weekly for 3 weeks out of 4 , with dose reductions allowed for toxicity. IMM-101 $\left(0.1 \mathrm{ml}\right.$ of $10 \mathrm{mg} \mathrm{ml}^{-1}$ suspension) was administered by intradermal injection into the skin overlying the deltoid muscle with the arm alternated for each dose. This dose of IMM-101 was previously shown to be safe and well tolerated in patients with melanoma (Stebbing et al, 2012). Dosing delays or half doses were allowed if skin reaction was unacceptable. IMM-101 was administered every 2 weeks for three doses followed by 4 weeks rest, then every 2 weeks for a further three doses. Subsequent doses were administered every 4 weeks; the first IMM-101 dose was administered 2 weeks before the first dose of GEM. Upon disease progression or toxicity to GEM, second-line chemotherapy of the investigator's choice was allowed.

Maximum treatment duration was 12 cycles. All patients who completed the study (from both treatment groups) were able to enter a long-term follow-up study in which all would receive IMM-101.

Assessments. Adverse events (AEs) were reported at each visit according to National Cancer Institute Common Terminology Criteria for Adverse Events version 4.0 (DCTD, NHI, DHHS, Bethesda, MD, USA; http://cstep.cancer.gov/reporting/ctc.html). To assess the impact on toxicity of the time on study treatment, the rates of patients per month on study reporting at least one $\mathrm{AE}$ or at least one serious $\mathrm{AE}$ (SAE) were calculated. Injection site reactions to IMM-101 were recorded at each visit and included assessments of pain, induration, wet drainage, erythema, and tenderness as well as any impact on daily activities. Tumour response was determined by investigator assessment at baseline, weeks 13, 25, 37, and 48 , and as clinically indicated according to RECIST v1.1 (Brussels, Belgium; http://www.eortc.be/recist). Complete (CR) or partial responses (PR), and stable disease beyond 3 months were confirmed by a second radiologist.

Statistical analysis. As an exploratory, phase II, POC study with safety and tolerability assessment as the primary endpoint, the trial was not formally sized to test a specific efficacy hypothesis. A target of 90 patients (on a 2:1 allocation basis) was considered feasible and sufficient to address the primary endpoint. This number of patients is broadly in line with the size of other randomised, phase II trials in oncology, and was considered sufficient to provide insight into the potential efficacy of IMM-101 on additional endpoints including OS, PFS, and ORR.

Safety assessment was based on frequency and incidence of AEs using the safety analysis set of all patients who received study drug. OS and PFS outcomes were displayed as Kaplan-Meier curves for the intent-to-treat (ITT) analysis set and for the metastatic and locally advanced subgroups (only a selection of Kaplan-Meier curves will be displayed in this paper). Median survival estimates as well as $95 \%$ confidence intervals (CI) were reported for each group. 
OS and PFS differences were tested by two-sided log-rank tests. Cox proportional hazard $(\mathrm{PH})$ regression models were used to estimate hazard ratios (HR) with $95 \%$ CI. Data were censored if patients remained alive (OS), or had no recorded progression (PFS) at the time of analysis, or were lost to follow-up. Survival times were calculated from the date of randomisation until death. PFS was defined as the interval between randomisation and radiological and/or clinical progression or death. ORR was defined as a complete or partial response. Disease stabilisation included those patients with a response and also stable disease $\geqslant 3$ months.

To assess the potential influence of baseline characteristics on survival and PFS outcomes, an exploratory multivariate stepwise analysis (Cox PH regression model) was conducted for factors reported as prognostic for survival in PDAC (carbohydrate antigen 19.9 (CA19.9), carcinoembryonic antigen (CEA), lactate dehydrogenase (LDH), CRP, neutrophil-lymphocyte ratio (NLR), total bilirubin, age, and PS) (Bilici, 2014; Haas et al, 2013) alongside treatment group for the ITT and metastatic subgroup.

\section{RESULTS}

Patients and treatment. Between July 2011 and August 2013, 110 patients were enrolled and randomised (Figure 1). The ITT analysis included all randomised patients. Five patients in the IMM-101 + GEM group and one patient in the GEM group remained alive and were censored for OS at the last point of follow up. A further six patients in the IMM-101 + GEM group and two patients in the GEM group were lost to follow-up and censored at the last allowed date of follow-up. Demographic and baseline characteristics were mostly balanced between the treatment groups, but with some differences observed for age distribution, gender, PS, time since diagnosis, CA19.9 and NLR (Table 1). 84\% of patients had metastatic disease on enrolment.

Safety and exposure. The Safety population was the same as the ITT population, except that one patient was excluded from the IMM-101 + GEM group, having been withdrawn before study drug administration.

Median time on study was 4.83 months (range $0.2-12.0$ ) for IMM-101 + GEM, and 2.79 months (0.5-10.9) for GEM. The total time on study was 414 months for the IMM-101 + GEM group and 133 months for the GEM group. Median duration of exposure to GEM was longer for IMM-101 + GEM compared to GEM (78 and 59 days, respectively). To assess the impact on toxicity of the longer time on study observed for the IMM-101 + GEM group, the rates of patients per month on study reporting at least one $\mathrm{AE}$ or at least one SAE were also calculated and are reported here.

Seventy three (99\%) patients reported at least one AE in the IMM-101 + GEM group compared with $35(100 \%)$ patients in the GEM group. The corresponding rate of patients reporting at least one AE per month on study for IMM-101 + GEM vs GEM was 0.18 vs 0.26 . Pyrexia occurred with the greatest difference in incidence between IMM-101 + GEM vs GEM (28.4\% v 8.6\%), with all cases in the IMM-101 + GEM group being grade 1 (majority) or grade 2. Grade 3 and higher AEs occurred in 57 (77\%) patients in the IMM-101 + GEM group and $26(74 \%)$ patients in the GEM group. All grade 3 and higher AEs with an incidence of $\geqslant 5 \%$ in either group are shown in Table 2.

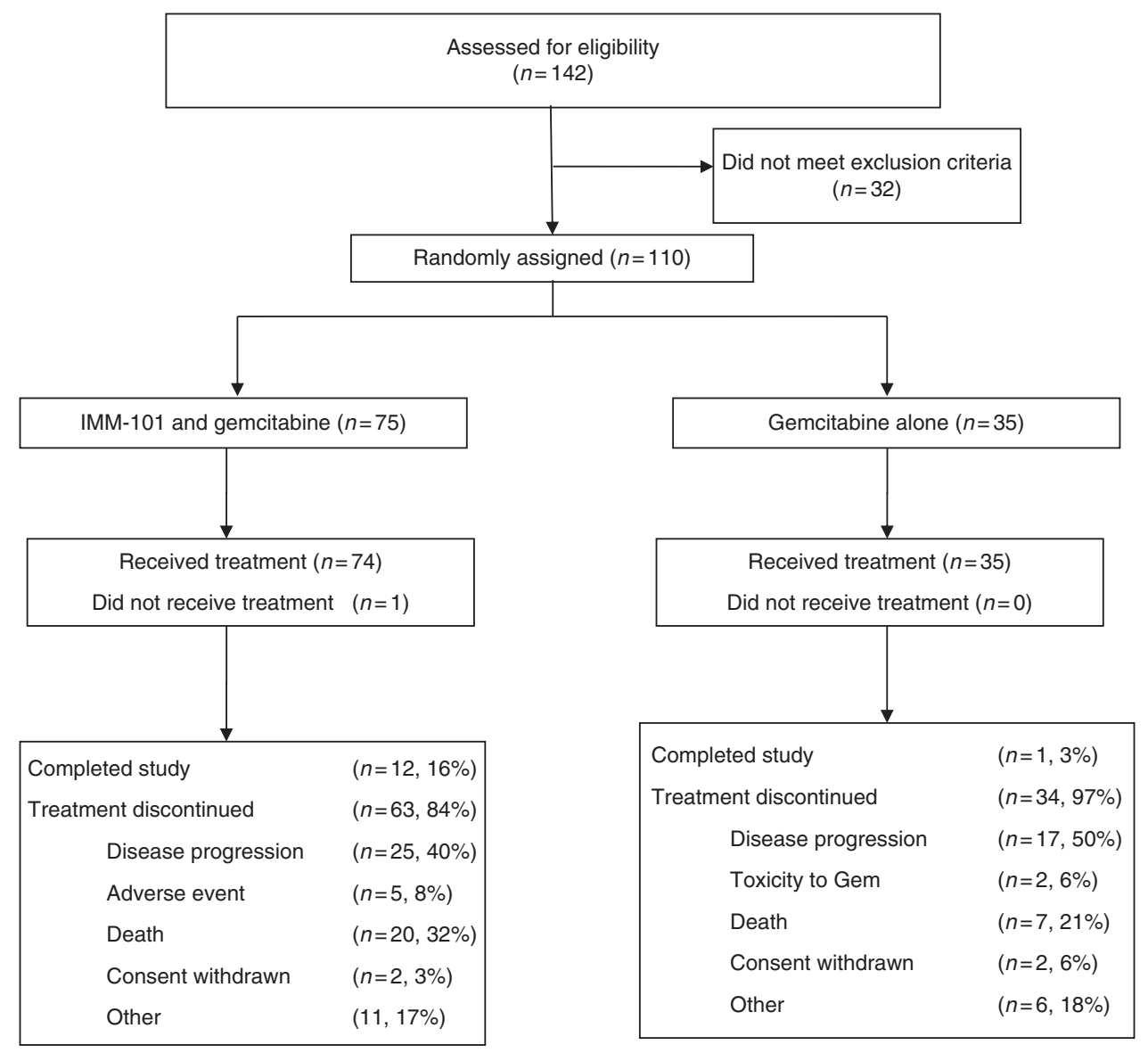

Percentages for reasons for withdrawal were calculated in relation to the total number of patients who discontinued treatment in each treatment arm

Figure 1. CONSORT diagram. 
Table 1. Baseline characteristics of all enrolled patients

\begin{tabular}{|c|c|c|}
\hline & $\begin{array}{c}\text { IMM-101+GEM } \\
(n=75)\end{array}$ & GEM $(n=35)$ \\
\hline \multicolumn{3}{|l|}{ Age, years } \\
\hline $\begin{array}{l}\text { Median } \\
\text { Range } \\
\text { Distribution, no (\%) } \\
\quad \leqslant 65 \\
>65\end{array}$ & $\begin{array}{c}68 \\
45-88 \\
\\
28(37) \\
47(63)\end{array}$ & $\begin{array}{c}66 \\
53-83 \\
16(46) \\
19(54)\end{array}$ \\
\hline \multicolumn{3}{|l|}{ Sex, no (\%) } \\
\hline $\begin{array}{l}\text { Female } \\
\text { Male }\end{array}$ & $\begin{array}{l}37(49) \\
38(51)\end{array}$ & $\begin{array}{l}14(40) \\
21(60)\end{array}$ \\
\hline \multicolumn{3}{|l|}{ Race, no (\%) } \\
\hline $\begin{array}{l}\text { White } \\
\text { Asian } \\
\text { Other } \\
\text { Unknown }\end{array}$ & $\begin{array}{c}74(99) \\
1(1) \\
0 \\
0\end{array}$ & $\begin{array}{c}33(94) \\
0 \\
1(3) \\
1(3)\end{array}$ \\
\hline
\end{tabular}

ECOG performance status (PS), no (\%)

\begin{tabular}{|l|c|c|}
\hline $0-1$ & $62(83)$ & $32(91)$ \\
2 & $13(17)$ & $3(9)$ \\
\hline
\end{tabular}

Time since first diagnosis, months

\begin{tabular}{|l|c|c|}
\hline Median & 1.22 & 0.76 \\
Range & $0.1-6.9$ & $0.1-3.9$
\end{tabular}

Extent of disease, no (\%)

\begin{tabular}{|c|c|c|}
\hline $\begin{array}{l}\text { Locally advanced } \\
\text { Metastatic }\end{array}$ & $\begin{array}{l}11(15) \\
64(85)\end{array}$ & $\begin{array}{r}7(20) \\
28(80)\end{array}$ \\
\hline \multicolumn{3}{|l|}{ CA19.9, $\mathrm{KUI}^{-1}$} \\
\hline $\begin{array}{l}\text { Median } \\
\text { Range } \\
\text { Distribution, no (\%) } \\
\quad \leqslant 1000 \\
\quad>1000\end{array}$ & $\begin{array}{c}485.8 \\
0.6-455,480 \\
38(58) \\
27(42)\end{array}$ & $\begin{array}{c}2747 \\
0.1-100000\end{array}$ \\
\hline
\end{tabular}

CEA, $\mu \mathrm{gl}^{-1}$

\begin{tabular}{|l|c|c|}
\hline Median & 10 & 9.3 \\
Range & $0.7-679.9$ & $2.0-681.0$ \\
$\begin{array}{l}\text { Distribution, no (\%) } \\
\quad \leqslant 10\end{array}$ & $33(51)$ & $17(55)$ \\
$>10$ & $32(49)$ & $14(45)$ \\
\hline
\end{tabular}

\section{$\mathrm{LDH}, \mathrm{UI}^{-1}$}

Median

Range

Distribution, no (\%)

$\leqslant 250$

$>250$

$32(49)$

$14(45)$

\begin{tabular}{|c|c|c|}
\hline & & \\
\hline \multicolumn{3}{|l|}{$\mathrm{CRP}, \mathrm{mgl}^{-1}$} \\
\hline Median & 10 & 11.9 \\
\hline Range & $0.8-330.0$ & $0.6-76.8$ \\
\hline \multicolumn{3}{|l|}{ Distribution, no (\%) } \\
\hline$\leqslant 10$ & $37(51)$ & $16(46)$ \\
\hline$>10$ & $35(49)$ & $19(54)$ \\
\hline \multicolumn{3}{|c|}{ Total bilirubin, $\mathrm{mg} \mathrm{dl}^{-1}$} \\
\hline Median & 0.6 & 0.68 \\
\hline Range & $0.24-4.40$ & $0.24-2.04$ \\
\hline \multicolumn{3}{|l|}{ Distribution, no (\%) } \\
\hline$\leqslant 1$ & $52(69)$ & $21(62)$ \\
\hline$>1$ & $23(31)$ & $13(38)$ \\
\hline \multicolumn{3}{|l|}{ NLR } \\
\hline Median & 3.42 & 3.91 \\
\hline Range & $1.24-1518.52$ & $0.53-9.11$ \\
\hline \multicolumn{3}{|l|}{ Distribution, no (\%) } \\
\hline$\leqslant 5$ & $55(73)$ & $28(80)$ \\
\hline$>5$ & $20(27)$ & $7(20)$ \\
\hline
\end{tabular}

Abbreviations: CA19.9 = carbohydrate antigen 19.9; CEA = carcinoembryonic antigen; $\mathrm{CRP}=\mathrm{C}$-reactive protein; $\mathrm{ECOG}=$ Eastern Cooperative Oncology Group; $\mathrm{GEM}=$ gemcitabine; $\mathrm{LDH}=$ lactate dehydrogenase, $\mathrm{NLR}=$ neutrophil-lymphocyte ratio.
Thirty six patients reported at least one SAE in the IMM$101+$ GEM group compared to 10 patients in the GEM group. The corresponding rate of patients reporting at least one SAE per month on study for IMM-101 + GEM vs GEM was 0.09 vs 0.08 . SAEs which occurred in $\geqslant 5 \%$ of IMM-101 + GEM treated patients were biliary sepsis, abdominal pain and pyrexia (each occurring in $5 \%$ of patients) and disease progression in the GEM group (6\%). The incidence of individual SAEs was low and no trend could be observed.

Fatal (grade 5) AEs were reported in 19\% of the IMM-101+ GEM group vs 14\% in GEM, although none were considered related to study drug.

IMM-101 injection site reactions were almost all mild or moderate with isolated severe reactions in $4 \%$ of patients who all subsequently continued treatment. Only 2 patients (3\%) required a reduction to half dose as a result of local reactions, and both completed the study. The worst impact of IMM-101 injection on patients' daily activities related to $8 \%$ reporting a moderate impact which resolved during their time on study.

Treatment-related AEs leading to withdrawal from study were reported in 5\% of the IMM-101 + GEM group (all but 1 event related to GEM and two also related to IMM-101) vs none in GEM.

Efficacy. Survival analysis of the ITT group included deaths in $85 \%$ of the IMM-101 + GEM group and $91 \%$ of the GEM group, with median follow-up of 6.7 months (range 0.4-30.3) and 4.9 months (0.5-16.8), respectively. Table 3 and Figure 2 show the results for median OS and PFS.

Median OS in the ITT population was 6.7 months for IMM$101+$ GEM v 5.6 months for GEM (HR, 0.68; 95\% CI, 0.44-1.04, $P=0.074)$; the difference was not formally statistically significant. $28 \%$ of patients in the IMM-101 + GEM group and $34 \%$ in the GEM group took second-line therapy; 15 patients from the IMM$101+$ GEM group continued to receive IMM-101.

Analysis of the predefined metastatic subgroup $(n=92)$ showed a difference in survival between the treatment groups with median survival for IMM-101 + GEM of 7.0 vs 4.4 months for GEM (HR, 0.54; 95\% CI $0.33-0.87, P=0.01$ ). The smaller subgroup of patients with locally advanced disease $(n=18)$ showed a lower median survival for IMM-101 + GEM of 6.7 months $v s 9.2$ months for GEM (HR, 3.81; 95\% CI 1.03-14.05, $P=0.032)$. Results are difficult to interpret in the latter subgroup because of the low number of patients.

The planned survival analysis based on PS (0-1 vs 2) was not performed because the PS2 subgroup from the GEM arm contained only thre patients.

Results for PFS reflected those for OS. In the ITT population, median PFS was 4.1 months for IMM-101+ GEM vs 2.4 months for GEM (HR, 0.58; 95\% CI 0.37-0.91; $P=0.016$ ); the difference was statistically significant. For the metastatic subgroup, median PFS was 4.4 months for IMM-101 + GEM vs 2.3 months for GEM (HR $0.46 ; 95 \%$ CI $0.28-0.75 ; P=0.001$ ). The small subgroup with locally advanced disease had median PFS of 3.4 months for IMM$101+$ GEM vs 5.3 months for GEM (HR 2.38; 95\% CI 0.65-8.78; $P=0.177)$.

The exploratory multivariate analysis of PFS and OS outcomes indicated that baseline CA19.9, CEA, CRP, NLR and randomised treatment were prognostic for PFS outcome in the ITT population and metastatic subgroup and also for OS outcome in the metastatic subgroup. For OS in the ITT population, randomised treatment fell marginally short of the multivariate stepwise inclusion criteria. Overall, these exploratory analyses confirmed that the differences seen for PFS and OS between IMM-101 + GEM v GEM were not attributable to important prognostic factors and any associated chance baseline imbalances. An exploratory multivariate analysis was not performed on the subgroup with locally advanced disease because the number of patients was too small. 
Table 2. Grade 3 and higher adverse events occurring in at least $5 \%$ patients in either group

\begin{tabular}{|l|c|c|c|}
\cline { 2 - 3 } & \multicolumn{2}{c|}{ Number of patients (\%) } \\
\hline NCI CTC adverse events & IMM-101 + GEM ( $\mathbf{n}=\mathbf{7 4})$ & GEM ( $\mathbf{n}=35)$ & $\begin{array}{c}\text { Difference in Incidence Rates } \\
\text { (IMM-101 + GEM-GEM) }\end{array}$ \\
\hline Asthenia & $8(11 \%)$ & $1(3 \%)$ & $8 \%$ \\
\hline Abdominal pain & $6(8 \%)$ & $1(3 \%)$ & $5 \%$ \\
\hline Vomiting & $4(5 \%)$ & 0 & $5 \%$ \\
\hline Anaemia & $6(8 \%)$ & $1(3 \%)$ & $5 \%$ \\
\hline Biliary sepsis & $4(5 \%)$ & 0 & $5 \%$ \\
\hline Bile duct obstruction & $4(5 \%)$ & $1(3 \%)$ & $2 \%$ \\
\hline Neutropenia and/or neutrophil count decreased & $13(18 \%)$ & $6(17 \%)$ & $1 \%$ \\
\hline Leukopenia and/or WBC count decreased & $3(4 \%)$ & $4(11 \%)$ & $-7 \%$ \\
\hline Hypokalaemia and/or blood potassium decreased & 0 & $2(6 \%)$ & $-6 \%$ \\
\hline Fatigue & $4(5 \%)$ & $4(11 \%)$ & $-6 \%$ \\
\hline Urinary tract infection & $1(1 \%)$ & $2(6 \%)$ & $-5 \%$ \\
\hline Disease progression & $3(4 \%)$ & $3(9 \%)$ & $-5 \%$ \\
\hline Thrombocytopenia and/or platelet count decreased & $5(7 \%)$ & $3(9 \%)$ & $-2 \%$ \\
\hline ALT increased & $3(4 \%)$ & $2(6 \%)$ & $-2 \%$ \\
\hline Abbreviations: ALT =alanine transaminase; GEM=gemcitabine; NCI CTC=National Cancer Institute Common Terminology Criteria; WBC= white blood cells. \\
\hline
\end{tabular}

Table 3. Overall survival and progression-free survival

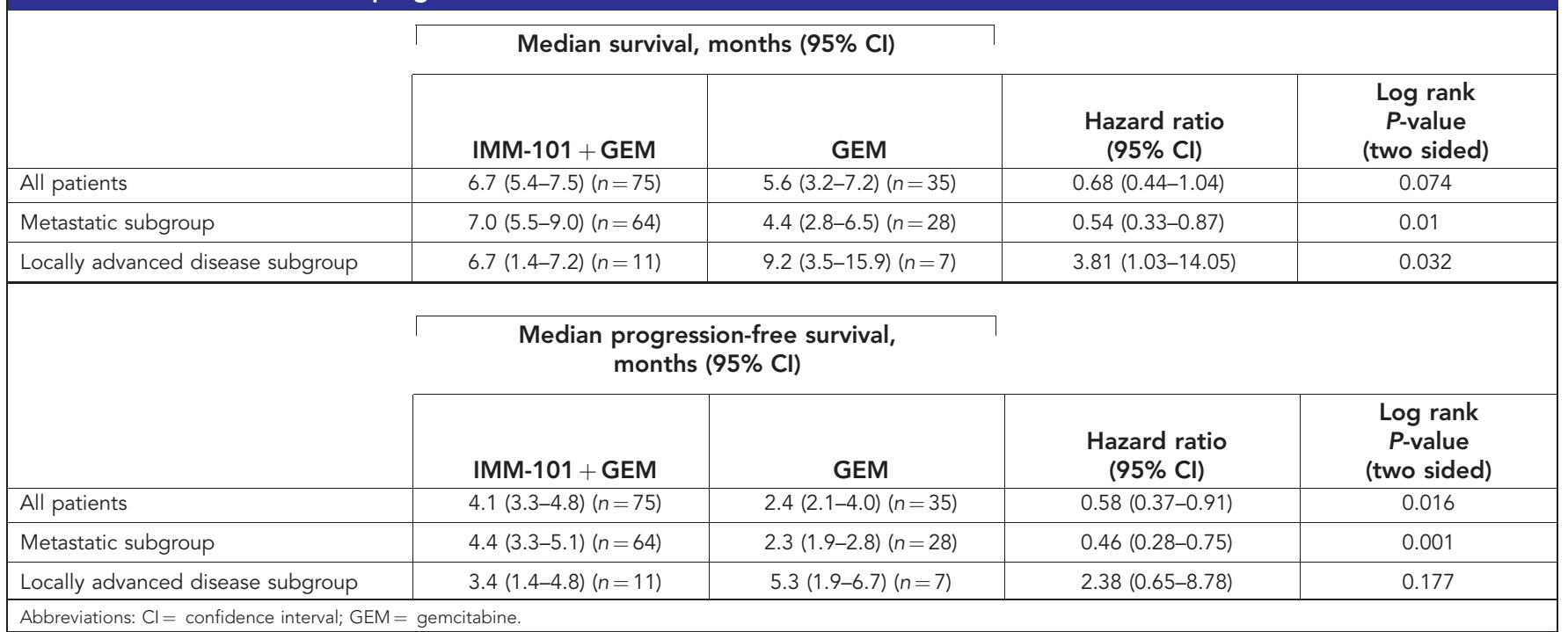

The ORR of $10.7 \%$ was numerically higher for IMM-101+ GEM (95\% CI 4.7-19.9) v 2.9\% for GEM (95\% CI 0.1-14.9; $P=0.164)$. The best overall response was a PR (i.e., there were no complete responders).

The disease stabilisation rate was $44 \%$ (95\% CI 32.5-55.9) for IMM-101 + GEM and 34.3\% (95\% CI 19.1-52.2, $P=0.334)$ for GEM.

\section{DISCUSSION}

IMAGE-1 is the first, randomised, phase II, POC study to explore the safety and tolerability of IMM-101 in combination with GEM vs GEM alone as first-line treatment in advanced PDAC. IMM$101+$ GEM was as well tolerated as GEM alone: the rates of patients per month on study reporting at least one $\mathrm{AE}$ or at least one SAE were similar between treatment groups.

Pyrexia is typical post-vaccination, and was more frequent in the IMM-101 + GEM group, with all cases classified as Grade 1 or 2. Injection-site reactions are a predictable reaction to mycobacterial antigens and were well tolerated by patients.

Grade 3 and higher AEs occurred at a similar incidence between treatment arms. No treatment-related deaths occurred.

This POC study was not formally sized to test a specific efficacy hypothesis, nonetheless, it has provided important insights into the potential for efficacy improvements with the use of IMM-101 in PDAC. For the overall ITT population, the median OS was similar between treatment groups while PFS was greater in the IMM$101+$ GEM group.

The pre-planned subgroup analysis of patients with locally advanced disease contained only $16 \%$ of the ITT population; 
A

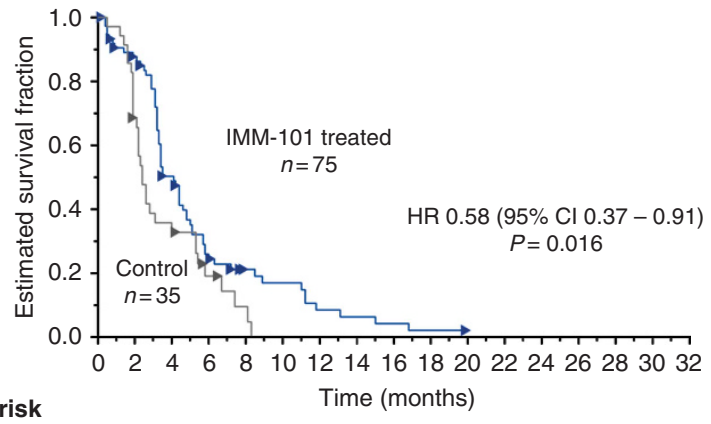

Number at risk

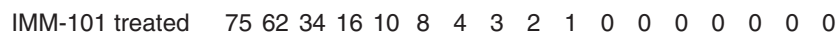
Control

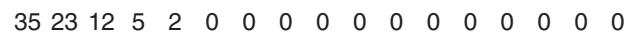

B

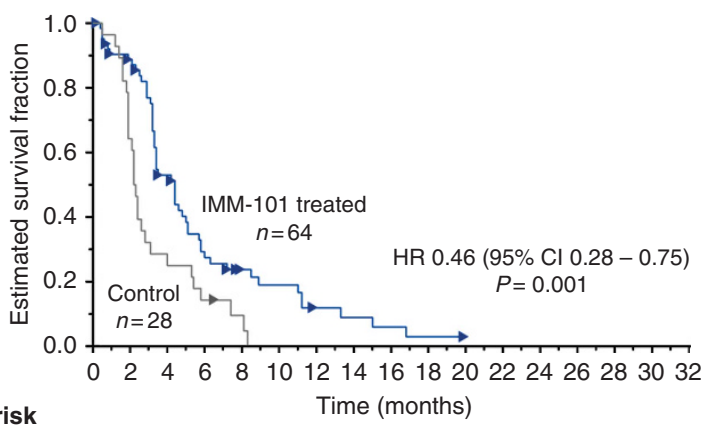

\section{Number at risk}

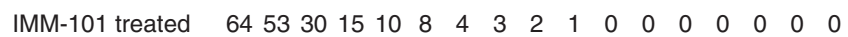
Control

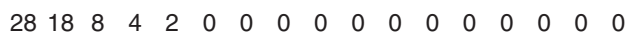

C

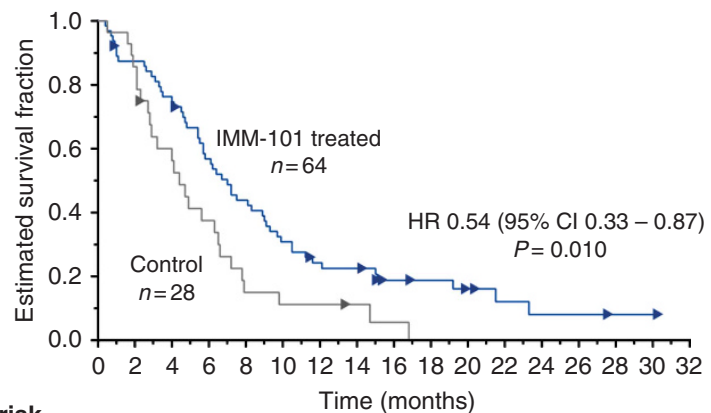

\section{Number at risk}

IMM-101 treated

Control

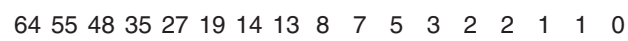

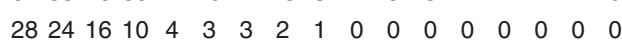

Figure 2. Progression-free survival ITT Analysis Set (A) and ITT Metastatic Subgroup (B). Overall survival Metastatic Subgroup (C). Arrows denote censored events. IMM-101 treated: IMM-101 + GEM; Control: GEM alone.

$n=11$ for IMM-101 + GEM and $n=7$ for GEM with just 11 and 6 deaths, respectively. Consequently, analyses in locally advanced patients lack robustness and are associated with wide CIs. In contrast, the pre-planned subgroup analysis of patients with metastatic disease contained $84 \%$ of the ITT population, and therefore, provide more reliable evidence regarding the possible effect of IMM-101 + GEM v GEM in this population. HRs and median OS and PFS were higher in the IMM-101 + GEM group, and associated CIs were narrower so increasing the confidence of the possibility of a true survival benefit in the IMM-101 + GEM group.

In a study of this size, with $2: 1$ randomisation, there is the chance that any apparent treatment benefit may be due to an imbalance in baseline characteristics, and some degree of imbalance for certain factors was noted (Table 1). However, a multivariate analysis based on factors reported as prognostic for survival in the literature (Bilici, 2014; Haas et al, 2013), and for which data were collected, showed, overall, that the differences seen for PFS and OS between the IMM-101 + GEM group vs GEM were not attributable to important prognostic factors and any associated chance baseline imbalances. Missing data from some patients (maximum of 15\% in IMM-101 + GEM group and 14\% in the GEM group) may have influenced results. Data were not collected on the site of primary tumour (head/body) which has been shown to be prognostic in some studies, although without a clear consensus (Bilici, 2014).

The median survival times for the GEM group were relatively low compared with some published information for pancreatic cancer patients receiving GEM monotherapy. The younger age of the study population in recently published phase III studies (58\% < age 65 years in MPACT (Von Hoff et al, 2013) and 71\% $\leqslant$ age 65 years in FOLFIRINOX trial (Conroy et al, 2011)) may have contributed to improved OS in those studies, although age was not shown to be prognostic for survival in IMAGE-1 which had $60 \%$ of patients aged over 65 years. Published median OS for patients receiving GEM monotherapy shows considerable variation from 4.9 months (Poplin et al, 2009) to 8.3 months (Colucci et al, 2010) (for predominantly metastatic populations). In a systematic review, median survival for patients with pancreatic cancer who underwent interventions (including chemotherapy, radiotherapy or surgery) ranged from 2-8.1 months (Carrato et al, 2015). In IMAGE-1, the relatively long time from diagnosis (Table 1) with eligibility not restricted to newly diagnosed patients, may have reduced median OS in both arms.

Upon disease progression or toxicity to GEM, the protocol allowed treatment changes to be made on study, rather than only after withdrawal. This facilitated data collection and allowed patients from the IMM-101 + GEM group to continue to receive IMM-101. The use of second-line anticancer therapy was balanced between treatment groups with $28 \%$ of patients in the IMM$101+$ GEM group and 34\% in the GEM group; this had no bearing on the ITT analysis.

In this study, IMM-101 in combination with GEM was as safe and well tolerated as GEM alone in patients with advanced PDAC, and there was a suggestion of a beneficial effect on survival in patients with metastatic disease. This supports further evaluation of IMM-101 in an adequately powered confirmatory study. Moreover, ongoing analyses to identify potentially predictive markers of response will guide the design of this new study.

Currently there are 150 studies listed on https://clinicaltrials.gov/ that are investigating the combination of GEM with nabpaclitaxel in different combination regimens for pancreatic cancer. Where reported, the study populations are significantly younger and clinically fitter than in IMAGE-1.

There are also several studies evaluating combinations with FOLFIRINOX. While promising activity has been seen as judged by response rate (Nywening et al, 2016), the toxicity of FOLFIRINOX-based regimens precludes treatment of the majority of patients with advanced pancreatic cancer.

The clinical crux is that GEM still represents the clinical option for patients with poorer performance status in this disease. In addition, single agent GEM still continues to be the standard of care for patients who are not fit for FOLFIRINOX in economically restricted health care systems globally. Therefore, our intention is to plan a large, adequately powered, phase III study of IMM-101 in combination with GEM for the first-line treatment of patients with metastatic pancreatic cancer. The patient population that is eligible for this study will be defined carefully to ensure that only those patients who are not suitable for treatment with GEM + nabpaclitaxel or FOLFIRINOX are enroled.

We do acknowledge the need to investigate IMM-101 in combination with standard of care in first-line treatment of metastatic pancreatic cancer, and also in second-line treatment of 
metastatic pancreatic cancer in combination with MM-398, a nanoliposomal encapsulation of irinotecan, +5 -fluorouracil and folinic acid (Wang-Gillam et al, 2016). Therefore, a follow-up phase I/IIa trial to evaluate the safety, tolerability and activity of IMM-101 in combination with different chemotherapy regimens in pancreatic cancer is currently being instigated.

\section{ACKNOWLEDGEMENTS}

This work was supported by Immodulon Therapeutics Ltd, 6-9 The Square, Stockley Park, Uxbridge, UB11 1FW, UK. European Clinical Trials Database (EudraCT) no. 2010-022757-42.

\section{CONFLICT OF INTEREST}

Angus G Dalgleish and Andrew Gaya have received travel grants and conference funding from Immodulon Therapeutics. Satvinder Mudan is an unsalaried director and shareholder of Immodulon Therapeutics, Ltd. Robert Glynne-Jones received honoraria and research funding from Merck KgaA, Roche and Sanofi-Aventis. Shama Wagle and Kevin Carroll, of TranScrip, LLP, provided medical writing and statistical support to Immodulon Therapeutics. The remaining authors declare no conflict of interest.

\section{AUTHOR CONTRIBUTORS}

Conception and design: Angus G Dalgleish, Justin Stebbing and Satvinder Mudan. Acquisition of data: Douglas J A Adamson, Justin Stebbing, Andrew Gaya, Seema Safia Arif, Paolo Bidoli, David Chang, Sue Cheeseman, Robert Diaz-Beveridge, Carlos Fernandez-Martos, Cristina Granetto, Rob Glynne-Jones, Bartomeu Massuti, Karen McAdam, Raymond McDermott, Andrés J Muñoz Martín, Demetris Papamichael, Roberto Pazo-Cid, Jose M Vieitez and Alberto Zaniboni. Data analysis and interpretation: Angus G Dalgleish. Manuscript writing: all authors. Final approval of manuscript: all authors.

Presented in part at the American Society of Gastrointestinal Cancers Symposium, San Francisco, January 2015, The American Society of Cancer Annual Symposium, Chicago, May 2015 and the European Society of Clinical Oncology, Barcelona, June 2015.

\section{REFERENCES}

Bazzi S, Modjtahedi H, Mudan S, Akle C, Bahr GM (2015) Analysis of the immunomodulatory properties of two heat-killed mycobacterial preparations in a human whole blood model. Immunobiology 220: 1293-1304.

Bilici A (2014) Prognostic factors related with survival in patients with pancreatic adenocarcinoma. World J Gastroenterol 20(31): 10802-10812.

Braiteh F, Patel M, Parisi M, Quanhong N, Park S, Faria C (2016) Comparative effectiveness and resource utilization of nab-paclitaxel plus gemcitabine (nab-P $+\mathrm{G}$ ) versus FOLFIRINOX (FFX) in first-line treatment of advanced pancreatic adenocarcinoma (PDAC) in a U.S. community oncology setting. J Clin Oncol 2016(4): abstract 433 .

Burris 3rd HA, Moore MJ, Andersen J, Green MR, Rothenberg ML, Modiano MR, Cripps MC, Portenoy RK, Storniolo AM, Tarassoff P, Nelson R, Dorr FA, Stephens CD, Von Hoff DD (1997) Improvements in survival and clinical benefit with gemcitabine as first-line therapy for patients with advanced pancreas cancer: a randomized trial. J Clin Oncol 15(6): 2403-2413.

Carrato A, Falcone A, Ducreux M, Valle JW, Parnaby A, Djazouli K, AlnwickAllu K, Hutchings A, Palaska C, Parthenaki I (2015) A systematic review of the burden of pancreatic cancer in Europe: real world impact on survival, quality of life and costs. J Gastrointest Cancer 46: 201-211.
Colucci G, Labianca R, Di Costanzo F, Gebbia V, Carteni G, Massidda B, Dapretto E, Manzione L, Piazza E, Sannicolo M, Ciaparrone M, Cavanna L, Giuliani F, Maiello E, Testa A, Pederzoli P, Falconi M, Gallo C, Di Maio M, Perrone F, Gruppo Oncologico Italia M. Gruppo Italiano per lo Studio dei Carcinomi dell'Apparato D, Gruppo Oncologico Italiano di Ricerca C (2010) Randomized phase III trial of gemcitabine plus cisplatin compared with single-agent gemcitabine as first-line treatment of patients with advanced pancreatic cancer: the GIP-1 study. J Clin Oncol 28(10): $1645-1651$.

Conroy T, Desseigne F, Ychou M, Bouche O, Guimbaud R, Becouarn Y, Adenis A, Raoul JL, Gourgou-Bourgade S, de la Fouchardiere C, Bennouna J, Bachet JB, Khemissa-Akouz F, Pere-Verge D, Delbaldo C, Assenat E, Chauffert B, Michel P, Montoto-Grillot C, Ducreux M. Groupe Tumeurs Digestives of U, Intergroup P (2011) FOLFIRINOX versus gemcitabine for metastatic pancreatic cancer. N Engl J Med 364(19): $1817-1825$.

Fowler D, Copier J, Wilson N, Dalgleish AG, Bodman-Smith MD (2011) Mycobacteria activate gamma delta T-cell anti-tumour responses via cytokines from type 1 myeloid dendritic cells: a mechanism of action for cancer immunotherapy. Cancer Immunol Immunother 61(4): 535-547.

Gunturu KS, Rossi GR, Saif MW (2013) Immunotherapy updates in pancreatic cancer: are we there yet? Therap Adv Med Oncol 5(1): 81-89.

Haas M, Heinemann V, Kullmann F, Laubender RP, Klose C, Bruns CJ, Holdenrieder S, Modest DP, Schulz C, Boeck S (2013) Prognostic value of CA 19-9, CEA, CRP, LDH and bilirubin levels in locally advanced and metastatic pancreatic cancer: results from a multicenter, pooled analysis of patients receiving palliative chemotherapy. J Cancer Res Clin Oncol 139(4): 681-689.

Hidalgo M, Cascinu S, Kleeff J, Labianca R, Lohr JM, Neoptolemos J, Real FX, Van Laethem JL, Heinemann V (2015) Addressing the challenges of pancreatic cancer: future directions for improving outcomes. Pancreatology 15(1): 8-18.

Le DT, Wang-Gillam A, Picozzi V, Greten TF, Crocenzi T, Springett G, Morse M, Zeh H, Cohen D, Fine RL, Onners B, Uram JN, Laheru DA, Lutz ER, Solt S, Murphy AL, Skoble J, Lemmens E, Grous J, Dubensky Jr T, Brockstedt DG, Jaffee EM (2015) Safety and survival with GVAX pancreas prime and Listeria Monocytogenes-expressing mesothelin (CRS-207) boost vaccines for metastatic pancreatic cancer. J Clin Oncol 33(12): 1325-1333.

Network. NCC Pancreatic adenocarcinoma (Version 2.2015).

NICE (2001) Guidance on the use of gemcitabine for the treatment of pancreatic cancer.

NICE (2015) Paclitaxel as albumin-bound nanoparticles in combination with gemcitabine for previously untreated metastatic pancreatic cancer.

Nywening T, Wang-Gillam A, Sanford D, Belt B, Panni R, Cusworth B (2016) Targeting tumour-associated macrophages with CCR2 inhibition in combination with FOLFIRINOX in patients with borderline resectable and locally advanced pancreatic cancer: a single-centre, open-label, dosefinding, non-randomised, phase $1 \mathrm{~b}$ trial. Lancet Oncol 17(5): 651-662.

Pico de Coana Y, Choudhury A, Kiessling R (2015) Checkpoint blockade for cancer therapy: revitalizing a suppressed immune system. Trends Mol Med 21(8): 482-491.

Poplin E, Feng Y, Berlin J, Rothenberg ML, Hochster H, Mitchell E, Alberts S, O’Dwyer P, Haller D, Catalano P, Cella D, Benson 3rd AB (2009) Phase III, randomized study of gemcitabine and oxaliplatin versus gemcitabine (fixed-dose rate infusion) compared with gemcitabine (30-minute infusion) in patients with pancreatic carcinoma E6201: a trial of the Eastern Cooperative Oncology Group. J Clin Oncol 27(23): 3778-3785.

Seufferlein T, Bachet JB, Van Cutsem E, Rougier P. Group EGW (2012) Pancreatic adenocarcinoma: ESMO-ESDO Clinical Practice Guidelines for diagnosis, treatment and follow-up. Ann Oncol 23(Suppl 7): vii33-vii40.

Stebbing J, Dalgleish A, Gifford-Moore A, Martin A, Gleeson C, Wilson G, Brunet LR, Grange J, Mudan S (2012) An intra-patient placebo-controlled phase I trial to evaluate the safety and tolerability of intradermal IMM-101 in melanoma. Ann Oncol 23(5): 1314-1319.

Tempero MA, Arnoletti JP, Behrman SW, Ben-Josef E, Benson 3rd AB, Casper ES, Cohen SJ, Czito B, Ellenhorn JD, Hawkins WG, Herman J, Hoffman JP, Ko A, Komanduri S, Koong A, Ma WW, Malafa MP, Merchant NB, Mulvihill SJ, Muscarella 2nd P, Nakakura EK, Obando J, Pitman MB, Sasson AR, Tally A, Thayer SP, Whiting S, Wolff RA, Wolpin BM, Freedman-Cass DA, Shead DA. National Comprehensive Cancer N (2012) 
Pancreatic Adenocarcinoma, version 2.2012: featured updates to the NCCN Guidelines. J Natl Comprehens Cancer Netw 10(6): 703-713.

Von Hoff DD, Ervin T, Arena FP, Chiorean EG, Infante J, Moore M, Seay T, Tjulandin SA, Ma WW, Saleh MN, Harris M, Reni M, Dowden S, Laheru D, Bahary N, Ramanathan RK, Tabernero J, Hidalgo M, Goldstein D, Van Cutsem E, Wei X, Iglesias J, Renschler MF (2013) Increased survival in pancreatic cancer with nab-paclitaxel plus gemcitabine. $N$ Engl J Med 369(18): 1691-1703.

Wang-Gillam A, Li C, Bodoky G, Dean A, Shan Y, Jameson G, Macarulla T, Lee K, Cunningham D, Blanc J, Hubner R, Chiu C, Schwartsmann G, Siveke J, Braiteh F, Moyo V, Belanger B, Dhindsa N, Bayever E, Von Hoff DD, Chen L (2016) Nanoliposomal irinotecan with fluorouracil and folinic acid in metastatic pancreatic cancer after previous gemcitabine-based therapy (NAPOLI-1): a global, randomised, open-label, phase 3 trial. Lancet Oncol 387(10018): 545-547.

Wormann SM, Diakopoulos KN, Lesina M, Algul H (2014) The immune network in pancreatic cancer development and progression. Oncogene 33: 2956-2967.

cc) (i) This work is licensed under the Creative Commons Attribution 4.0 International License. To view a copy of this license, visit http://creativecommons.org/licenses/by/4.0/ 